\title{
PERMUTATION INEQUALITIES
}

\author{
JURIJ KOVIČ
}

Abstract. We formulate and solve some special cases of the following (in general NP-hard) extremal problem: "Given a graph $G$ (or a hypergraph $H$ ), label its vertices with given different $n$ non-negative numbers $a_{1} \geqslant a_{2} \geqslant \ldots \geqslant a_{n} \geqslant 0$ in such a way that the sum of the products of labels in adjacent vertices $f=\sum a_{i} a_{j}$ will be maximal (or minimal)". Solving this problem for some special families of graphs (e.g. paths, trees and stars) we obtain examples of "permutation inequalities" $f_{\min } \leqslant f \leqslant f_{\max }$.

Mathematics subject classification (2010): 05A20, 05C22, 05C78, 26D15.

Keywords and phrases: Inequality, permutation, rearrangement, bilinear function, multilinear function, labeled graph, hypergraph.

\section{REFERENCES}

[1] C. Berge, Hypergraphs, North-Holland Mathematical library, 45, Elsevier Science Publiching Company, INC., New York (1989).

[2] V. BERINDE, Exploring, Investigating and Discovering in Mathematics, Birkhäuser Verlag (2004).

[3] P. BRUIN, Proof of rearrangement inequality (version 1), PlanetMath.org. Freely available at http://planetmath.org/proof of rearrangementinequality.

[4] M. R. GAREY, D. S. Johnson, Computers and Intractability, W. H. Freeman (1979).

[5] G. H. Hardy, J. E. LitTlewood, G. Pólya, Inequalities, Cambridge University Press, Cambridge (1952).

[6] N. Hartsfiled, G. Ringel, Pearls in Graph Theory, Academic Press, San Diego (1994).

[7] C. Jordan, Sur les assemblages de lignes, Journal für die Reine und Angewandte Mathematik, 70 (1869), 185-190.

[8] E. L. LaWler, Combinatorial Optimization: Networks and Matroids, Holt, Rinehart and Winston, New York (1976).

[9] D. S. Mitrinović, Nejednakosti, Gradjevinska knjiga, Beograd (1965).

[10] Rearrangement inequality, en.wikipedia.org/wiki/Rearrangement_inequality. 